\title{
Comparing MTA and Ketac Molar Easymix for Furcation Perforation Repair using a Volumetric Method
}

\author{
Sadullah KAYA ${ }^{1}$, Selengül GANİDAĞLI AYAZ ${ }^{2}$, Mehmet Sinan DOĞAN ${ }^{3}$, Haluk AYDI N ${ }^{4}$ \\ ${ }^{\mathbf{1}}$ Dicle University, Faculty of Dentistry, Department of Operative Dentistry and Endodontics, Diyarbakir, Turkey \\ 2 Dicle University, Faculty of Dentistry, Department of Operative Dentistry and Endodontics, Diyarbakir, Turkey \\ 3 Dicle University, Faculty of Dentistry, Department of Pediatric Dentistry, Diyarbakir, Turkey \\ ${ }^{4}$ Dicle University, Science and Arts Faculty, Department of Chemistry, Diyarbakir, Turkey
}

\section{Key Words}

MTA, Ketac Molar Easymix, volume measurement method, methylene blue.

\section{Correspondence: \\ Sadullah KAYA \\ Dicle University, \\ Faculty of Dentistry, \\ Department of Operative Dentistry \\ and Endodontics, \\ 21280, Diyarbakir, TURKEY. \\ e-mail: sadullahkaya@hotmail.com}

\begin{abstract}
Aim: We compared the ability of mineral trioxide aggregate (MTA) and Ketac Molar Easymix (KM) to repair furcal perforations in extracted human molars, based on the volume of methylene blue dye penetration.

Methodology: In total, 44 human mandibular molars were divided randomly into two ( $n=20$ each) experimental groups, with two teeth used as positive controls and two teeth without perforations used as negative controls. Group 1 was repaired with MTA and group 2 with Ketac Molar Easymix. The volumetric determination of dye penetration was based on the molecular characteristics of methylene blue. The standard area of a methylene blue particle is known and the surface area can be calculated. We converted the dye penetration area into a volume and performed quantitative analyses.

Results: Volume measurement using the dye penetration method showed that KM resulted in more microleakage than MTA $(p<0.05)$. Conclusions: Mineral trioxide aggregate resulted in significantly less dye leakage than Ketac Molar Easymix using a volumetric measurement method.
\end{abstract}

(Int Dent Res 2011;1:13-17)

\section{Introduction}

Perforations of teeth are procedural accidents that can have adverse effects on the success of endodontic treatment. The etiology of dental perforations includes deep caries, resorption, or iatrogenic factors. Regardless of the cause, a perforation allows microorganisms to invade the supporting structures, triggering inflammation and a loss of attachment, which may ultimately compromise the prognosis of the tooth $(1,2)$. In a literature review, Alhadainy (3) stated the ideal features of a perforation repair material: it should be biocompatible, non-toxic, radiopaque, nonresorbable, and bacteriostatic, and have excellent sealing qualities. Additionally, the repair material should be esthetically pleasing. Mineral trioxide aggregate (MTA) is used for furcation repair, resorption treatment, pulpotomy procedures, and capping pulps with reversible pulpitis (4-6). However, one major disadvantages of using MTA is its long setting time (7).

Glass ionomer cements are popular in restorative dentistry because of their esthetic properties. Clinical studies have provided evidence of the effectiveness of Ketac Molar as a restorative (8). The adhesion of Ketac Molar Easymix (KM) to dental tissue relies primarily on a chemical interaction and to a lesser extent on micromechanical interlocking (9). Several leakage models have been used to assess the ability of 
materials to seal furcation perforations, including dye extraction, dye penetration, fluid filtration, and bacterial leakage models (10). One of the major problems when using dyes to examine penetration is the air trapped in voids along root canal fillings (11, 12). Dye extraction studies also use dye penetration. In this method, samples stained using $2 \%$ methylene blue are stored in a hermetically sealed vial containing $65 \%$ nitric acid for 3 days. The vials are centrifuged at high speed to separate the extracted dye from the soluble dental tissues. The supernatant from each sample is then analyzed in an ultraviolet-visible spectrophotometer with concentrated nitric acid as the blank $(10,13)$.

Using the dye-extraction method, Hamad et al. (14) compared the sealing ability of gray and white MTA when used for furcation perforation repair of teeth. No significant difference between gray and white MTA was observed.

The theory underlying the use of methylene blue to measure the microleakage surface area is based on its adsorption characteristics, which can be explained by the collection of molecules, atoms, or ions in any solution on the surface of a solid when it turns into the gas, vapor, or liquid phase (15). This method can be used to determine the mass or volume of methylene blue. In this technique, MB, which has a known molecular weight $(\mathrm{MA}=319.868 \mathrm{~g} \cdot \mathrm{mol}-1)$, wavelength $\quad(\lambda \max )$
$(662 \mathrm{~nm})$, color index (52015), and surface area ( $\sigma=120 \mathrm{A0.2}$ ), is used as the adsorbent (15).

In this study, we compared the ability of MTA and Ketac Molar Easymix to seal furcation perforations in mandibular molars using volume measurement with the dye penetration method.

\section{Materials and Methods}

Forty-four extracted, human mandibular molars were used. All teeth were stored in distilled water at $4^{\circ} \mathrm{C}$ until used. The molars were decoronated $3 \mathrm{~mm}$ above the cemento-enamel junction and the roots were amputated $3 \mathrm{~mm}$ below the furcation using a tapered diamond stone. A standardized endodontic access opening was made in each tooth, sticky wax was placed over the orifice of each canal, and the teeth, including the pulpal floor, were coated with three layers of red nail varnish. To ensure that each perforation was centered between the roots, a defect $2 \mathrm{~mm}$ in diameter was made from the external surface with a \#3 high-speed round bur (Fig. 1, A-B). The teeth were divided randomly into two groups and the perforations were sealed as follows:

Groups $1(n=20)$ repaired with MTA (Dentsply Tulsa Dental, Tulsa, OK), Groups $2(n=20)$ repaired with Ketac Molar Easymix (3M ESPE AG)-a
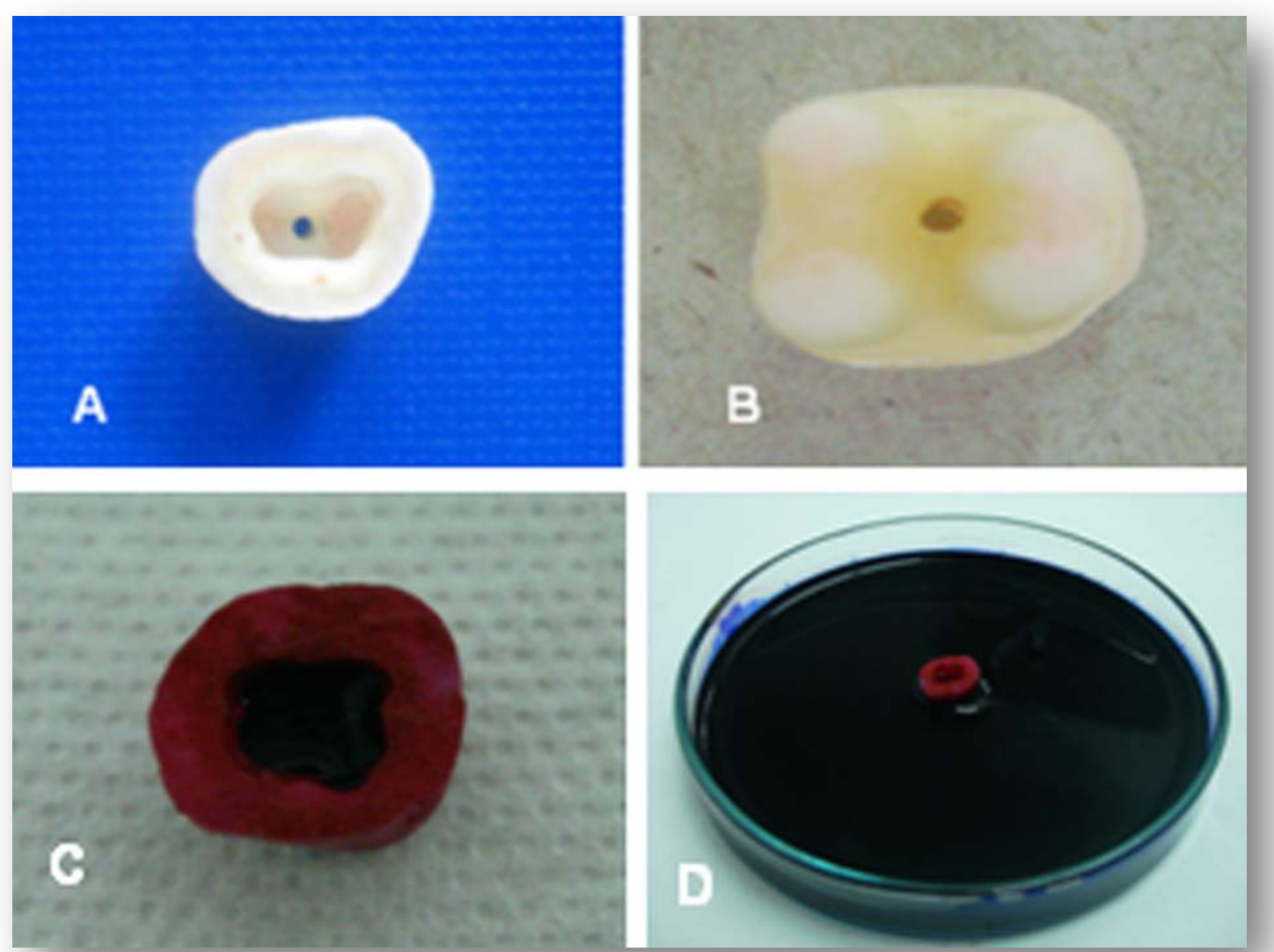

Figure 1. (A) Pulpal flor; and (B) furcal views of the perforation. A view of a tooth treated with methylene blue; (C) Orthograde direction; (D) retrograde direction. 
chemically cured glass ionomer cement. Two teeth with unrepaired perforations were used as positive controls and two teeth without perforations were used as negative controls. Teeth were stored for $24 \mathrm{~h}$ at $37^{\circ} \mathrm{C}$ and $100 \%$ humidity in an incubator to allow setting of MTA and KM. Each group was placed in petri dishes. All samples were stored in methylene blue for $72 \mathrm{~h}$ (Fig. 1, C-D). After removal from the dye, teeth were rinsed under tap water for $60 \mathrm{~min}$ and varnish removed with a polishing disc.

\section{Volume measurement method}

Samples $(n=40)$ were placed into two different glass vials containing $20 \mathrm{~mL}$ of concentrated $65 \%$ nitric acid until they all dissolved completely approximately for $72-h$. The MB that filled the microleakage gaps also dissolved in nitric acid. At the end of the adsorption period, the solution was centifugated for $20 \mathrm{~min}$ at $3000 \mathrm{rpm}$. After centrifugation, the dye concentration in the supernatant solutions was analyzed using a UV-spectrophotometer (Shimadzu UV-160, Shimadzu Corp., Kyoto, Japan) by monitoring the absorbance. The absorption wavelengths for different concentrations of MB were measured to construct the calibration graph (Fig. 2). With the help of the calibration graph, when the spectrophotometrically obtained absorption values were used, total concentrations were calculated by means of the

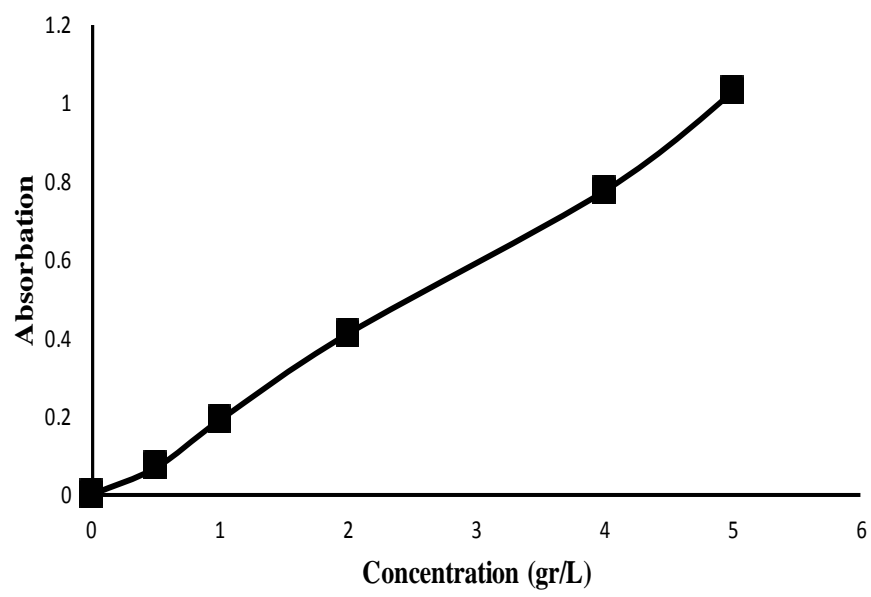

Figure 3. Calibration curve of methylene blue in different concentrations

\section{Discussion}

A furcation root perforation complicates endodontic treatment and compromises the prognosis if it is not repaired properly. It is crucial that the area of root perforation does not become infected, and the perforation should be repaired in a equation $A=(0.205 . C)-0.0139$. Concentration values were converted into volumetric values in $\mathrm{mm} 3$ using the mass and density values that were obtained via the calibration graph. Following the use of the equation $S=V M / M A . \sigma . N A$, where $S$ is surface area, VM is the single incremental capacity, MA is the molecular weight, $\sigma$ is the surface area of a single adsorbed particle, and NA is the Avogado constant (16), surface area is calculated, representing quantitative results.

The groups were compared statistically using the Mann-Whitney U-test. The comparison found a significant $(p<0.05)$ difference between the two groups.

\section{Results}

Microleakage results in $\mathrm{mm} 3$ are given in Table 1. The mean volumes of apical leakage values were $3.309 \times 10-4 \mathrm{~mm} 3$ for MTA, $4.915 \times 10-4 \mathrm{~mm} 3$ for KM. A significant statistically difference in leakage volume was found between the groups $(P<0.05)$. The positive controls demonstrated high apical microleakage (mean: 24.186×10-4 mm3; $P<0.001$ ), while no leakage was detected in the negative control group.

Table 1. Evaluation of the furcal leakage of MTA and $\mathrm{KM}$ with volume measurement technique.

Volume measurement

\begin{tabular}{lcc} 
Groups & No. teeth & $\left(\mathrm{mm}^{3} .10^{-3}\right)$ \\
& & Mean \pm SD \\
& 20 & $165 \pm 1.26^{\mathbf{a}}$ \\
\hline MTA & 20 & $245 \pm 1.17^{\mathbf{b}}$ \\
KM & \\
\hline & \\
a,b Groups with significant differences are shown with \\
supercripted letters.
\end{tabular}

timely manner if possible (17). Many restorative materials have been used to repair furcation perforations in dentistry (18). Various in vitro methods have been used to compare MTA with these materials (19-21). The majority of recently published data dealing with the use of MTA in root and furcal perforations is based on in vitro and in vivo studies (22).

Various leakage models have been used to assess the ability of materials to seal furcation perforations, including dye extraction, dye 
penetration, fluid filtration, and bacterial leakage models (14); there are advantages and disadvantages to each. In this study, we used a volume measurement method. Comparing the volume measurement method with fluid filtration and dye extraction methods, the former technique has the advantages of a shorter time requirement, greater accuracy, changes that are not simply dependent on spectrophotometric readings, and the ability to calculate small volume using devices that are standard equipment in most laboratories. Additionally, the volumetric measurement method gives quantitative results. The materials used are important and may affect the study results. MTA (17), amalgam (23), calcium hydroxide (24), and glass ionomer cements $(17,25)$ are among the materials that have been tested for repairing furcation perforations. The quality of the repairs demonstrates the difficulty in repairing furcal perforations. Many factors can affect the repair, including the technique used, the material chosen, the physician's ability, the clinical conditions, and the biocompatibility of the repair material $(17,26)$. Our found a significant $(p<0.05)$ difference between the MTA and KM groups.

Any material or technique may have particular features that must be considered with its clinical use. MTA is a fine powder, composed primarily of tricalcium silicate, tricalcium oxide, tricalcium aluminate, and silicate oxide, that forms a colloidal gel on hydration that solidifies in approximately $3 \mathrm{~h}$ (26). Consequently, when used as a root repair material, although there is some moisture on the external surface of the periradicular tissues, to assure proper setting, the internal aspect of the root must also be moistened using a cotton pellet.

\section{Conclusions}

Within the limitations of this study, MTA resulted in significantly less dye leakage than Ketac Molar Easymix using a volumetric measurement method. The volumetric method may be a good alternative for evaluating leakage because it includes most of the advantages of leakage studies and gives quantitative results.

\section{References}

1. American Association of Endodontists Glossary of Endodontic Terms. 2003; 7th ed; 2003.

2. Ruddle $\mathrm{C}$. Nonsurgical Endodontic Retreatment. In Cohen S, Burns RC (eds). Pathways of the pulp, 8th ed. St Louis: Mosby Inc., 2002:917.

3. Alhadainy HA. Root perforations. A review of literature. Oral Surg Oral Med Oral Pathol 1994; 78:368-74.

4. Tuna D, Olmez A. Clinical long-term evaluation of MTA as a direct pulp capping material in primary teeth. Int Endod J 2008;41:273-8.

5. Torabinejad M, Chivian N. Clinical applications of mineral trioxide aggregate. J Endod 1999;25: 197-205

6. Sarı S, Sönmez D. Internal resorption treated with mineral trioxide aggregate in a primary molar tooth: 18 month follow-up. J Endod 2006; 32:69-71.

7. Islam I, Chng HK, Yap AU. Comparison of the physical and mechanical properties of MTA and Portland cement. J Endod 2006; 32: 193-7.

8. Taifour D, Frencken JE, Beiruti N, van't Hof MA, Truin GJ. Effectiveness of glass-ionomer (ART) and amalgam restorations in the deciduous dentition: Results after 3 years. Caries Res 2002; 36: 437-44.

9. Glasspoole EA, Erickson RL, Davidson CL. Effect of surface treatments on the bond strength of glass ionomers to enamel. Dent Mater 2002; 8:454-62.

10. Camps J, Pashley D. Reliability of the dye penetration studies. J Endod 2003;29:592-4.

11. Barthel CR, Moshonov J, Shuping G, Orstavik D. Bacterial leakage versus dye leakage in obturated root canals. Int Endod J 1999;32:370-5.

12. Oliver CM, Abbott PV. Entrapped air and its effect on dye penetration of voids. Endod Dent Traumatol 1991; 7:135-8.

13. Hashem AAR, Hassanien EE. ProRoot MTA, MTAAngelus and IRM used to repair large furcation perforations: sealability study. J Endod 2008; 34:59-61.

14. Hamad HA, Tordik PA, McClanahan SB. Furcation perforation repair comparing gray and white MTA: a dye extraction study. J Endod 2006; 32:337-40.

15. Aydın H, Baysal G. Adsorption of acid dyes in aqueous solutions by shells of bittim (Pistacia khinju stocks), besalination. 2006; 196:248-59.

16. Pathomvanich S, Edmunds DH. The sealing ability of Thermafil obtutarors assessedby four different microleakage techniques. Int Endod J 1996;29:327-34.

17. Daoudi MF, Saunders WP. In Vitro Evaluation of Furcal Perforation Repair Using Mineral Trioxide Aggregate or Resin Modified Glass Ionomer Cement with and without the Use of the Operating Microscope. J Endod. 2002;28:512-5.

18. Bryan EB, Woollard G, Mitchell WC. Nonsurgical repair of furcal perforations: a literature review. Gen Dent 1999; 47:274-8. 
19. Nakata TT, Bae KS, Baumgartner JC. Perforation repair comparing mineral trioxide aggregate and amalgam using an anaerobic bacterial leakage model. J Endod 1998;24:184-6.

20. Hardy I, Liewehr FR, J oyce AP, Agee K, Pashley $\mathrm{DH}$. Sealing ability of One-Up Bond and MTA with and without a secondary seal as furcation perforation repair materials. J Endod 2004;30:658-61.

21. Hashem AA, Hassanien EE, ProRoot MTA. MTAAngelus and IRM used to repair large furcation perforations: sealability study. J Endod 2008;34:59-61.

22. Pace R , Giuliani V, Pagavino G. Mineral Trioxide Aggregate as Repair Material for Furcal Perforation: Case Series. J Endod 2008;34:11303.

23. Grossman LI. The management of accidents encountered in endodontic practice. Dent Clin North Am 1957;2:11.

24. Imura N, Mie Otani S, Hata G, Toda T, Zuolo ML. Sealing ability of composite resin placed over calcium hydroxide an calcium sulphate plugs in the repair furcation perforation in mandibular molars: a study in vitro. Int Endod J 1998;31:7984.

25. Fuss Z, Szajkis S, Tagger M. Periodontal response to glass ionomer cement in treatment of furcation perforations in dogs. J Dent Res 1992; 71: 1031.

26. Nicholls E. Treatment of traumatic perforations of the pulp cavity. Oral Surg Oral Med Oral Pathol 1962; 15:603-12. 